centration axis at $175 \mathrm{c} / \mathrm{min}$. , which agrees well with the measured background of $162 \mathrm{c} . / \mathrm{min}$., as it should. Another set of results for this series of tritiated water solutions was obtained with the water in the saturator maintained at $23 \cdot 6^{\circ} \mathrm{C}$. ; these, which are also plotted in Fig. 2, show much more seatter than do the results obtained at $0^{\circ} \mathrm{C}$., because the temperature was not maintained at exactly $23 \cdot 6^{\circ} \mathrm{C}$. but fluctuated about this value.

The results obtained demonstrate the feasibility of counting in the Geiger region at high relative humidities. They point to the almost certain possibility of absolute counting for tritium in the form of THO. The results also indicate the possibilities for the design of counters specifically to be used at high humidities. The paper by Cockroft and Curran ${ }^{6}$ is particularly suggestive in this light.

More complete and extensive results will be presented elsewhere as the work progresses. I wish to thank Dr. W. H. Johnson of Purdue University for his helpful comments.

Libbey-Owens-Ford Glass Co.,

EDWARd B. BuTler

Toledo 5, Ohio.

$$
\text { Aug. } 31 .
$$

${ }^{1}$ Pace, N., Kline, L., Schachman, H. K., and Hafenist, M., J. Biol. Chem., 168, 459 (1947).

'Wilzback K. E., Kaplan, L., and Brown, W. G., Science, 118, 522 (1953).

8 Wing, J., and Johnston, W. H., Science, 121, 674 (1955).

${ }^{4}$ White, D. F., Campbell, I. G., and Payne, P. R., Nature, 166, 628 (1950).

- Nilsson, G., and Aniansson, G., Nucleonics, 13, No. 2, 35 (1955).

- Cockroft, A. L., and Curran, S. C., Rev. Sci. Instr., 22, 37 (1951).

\section{Measurement of Tritium in Water Samples}

THE concentration of tritium in water samples has been measured by a new method which is both simple and rapid. The beta-rays of very low energy which are emitted by tritium are very difficult to count because of their inability to penetrate the windows of conventional Geiger counters. It has therefore been the practice for some time to introduce the tritium in various chemical combinations into the interior of a counter.

The method to be described consists of introducing the tritium in the form of water vapour as part of the filling of an internal gas counter. Any tritium atoms disintegrating inside the sensitive volume of the counter give rise to counts, and from the observed count-rate the number of tritium atoms present is evaluated.

The water vapour at saturation vapour pressure is introduced and the filling completed with 10-15 cm. pressure of a standard argon-alcohol mixture. An accurate measure of the pressure of the water vapour is obtained from the temperature and tables of saturation vapour pressures.

The argon-alcohol mixture is prepared beforehand in the normal ratio of 9 to 1 , and stored in a 5-litre glass bulb at an initial pressure of $30 \mathrm{~cm}$. Absolute alcohol is used and the argon is purified by flowing through a charcoal trap at liquid-nitrogen temperature.

Counters manufactured by Twentieth Century Electronics, type GA.10.M., are used. The outer envelope is made of glass and the cathode is of steel. The sensitive volume of the counter is about 50 c.c.

Using these counters in conjunction with an external quench unit, type $1014 A$, consistent plateaux are obtained with a length of more than $400 \mathrm{~V}$. and a slope less than 2 per cent per $100 \mathrm{~V}$. The threshold voltage is about 1,400 . The efficiency, as compared with a 10.cm. argon filling and using an external source of cobalt-60, is 98 per cent. The counters are not sensitive to light. If, after counting at a rate of 10,000 per min., the counter is pumped only for a few minutes, the residual contamination produces about 200 counts per min. This is easily removed by flushing two or three times with pure water vapour and if necessary heating slightly.

The normal working pressure of the vacuum apparatus is 1 micron, which can easily be obtained with a single-stage rotary vacuum pump and a liquid-nitrogen trap.

Isotope Division,

\section{J. F. Cameron}

Atomic Energy Research Establishment, Harwell, Didcot, Berks.

Aug. 29.

\section{The Reduction of Vitamin $\mathbf{B}_{12}$}

Two distinct reduction products of vitamin $B_{12}$ have been described in the literature, by Diehl and Murie ${ }^{1}$ and by Boos, Carr and Conn ${ }^{2}$; we shall refer to these as I and II respectively. They differ in their method of preparation, and have widely different absorption spectra in the visible and ultra-violet, but may both be re-oxidized to vitamin $\mathrm{B}_{12 a}$ by exposure to air, and from polarographic and titrimetric evidence both are claimed to contain divalent cobalt.

Compounds with spectra closely resembling that of I may be obtained by the action of a variety of reducing agents on vitamin $B_{12}$. Diehl and Murie employed catalytic hydrogenation; but cysteine in alkaline solution, lithium boron hydride, heating in 96 per cent ethanol for $12 \mathrm{hr}$. at $100^{\circ} \mathrm{C}$. in a nitrogen atmosphere, and chromous acetate in aqueous solu. tion, all give similar products, at least initially.

Hydrogenation, using palladium on barium sulphate as catalyst, readily continues beyond the stage represented by $I$, which in consequence cannot be obtained in a pure state by this method. For this reason, vitamin $\mathbf{B}_{12 a}$ is obtained in yields of only 60-70 per cent on re-oxidation (Boos et al. ${ }^{2}$; Brockman et $\left.a l .^{3}\right)$. Hydrogenation finally results in visually colourless products with ill-defined ultra-violet absorption; but intermediate compounds with diminished visible colour may also be obtained. On exposure to air, these give brown compounds with spectra somewhat similar to that of $I$, but which are altered by $p \mathrm{H}$ changes. They also form reddish cyanide derivatives. They are characterized by rather broad bands in the $435-450 \mathrm{~m} \mu$ region, and the benziminazole of the nucleotide is no longer coordinated to the cobalt, as indicated by the sensitivity ${ }^{4}$ of the spectra in the $280 \mathrm{~m} \mu$ region to $p \mathrm{H}$. A very similar sequence is obtained by reduction with lithium boron hydride in aqueous solution. Reduction with chromous acetate in aqueous solution ( $p H$ c. 5), however, was found to give a solution of I which was stable indefinitely in a hydrogen atmosphere, even in the presence of excess reducing agent. Its spectrum (see Fig. 1) is very similar to that given by Diehl and Murie, and re-oxidation to vitamin $B_{12 a}$ appears to be substantially quantitative.

Boos et al. obtained II by reduction of vitamin $B_{12}$ in ethylenediaminetetraacetate buffer at $p H \mathbf{H} 9.5$ with chromous chloride. They observed, however, that the product obtained by the addition of one equivalent 